QCD Evolution Workshop 2014

International Journal of Modern Physics: Conference Series

Vol. 37 (2015) 1560050 (12 pages)

(C) The Author

DOI: $10.1142 / \mathrm{S} 2010194515600502$

\title{
Virtuality Distributions and Pion Transition Form Factor*
}

\author{
A. V. Radyushkin \\ Physics Department, Old Dominion University, \\ Norfolk, VA 23529, USA \\ Thomas Jefferson National Accelerator Facility, \\ Newport News, VA 23606, USA \\ radyush@jlab.org
}

Published 25 February 2015

\begin{abstract}
Using the example of hard exclusive transition process $\gamma^{*} \gamma \rightarrow \pi^{0}$ at the handbag level, we outline basics of a new approach to transverse momentum dependence in hard processes. In coordinate representation, matrix elements of operators (in the simplest case, bilocal $\mathcal{O}(0, z))$ describing a hadron with momentum $p$, are functions of $(p z)$ and $z^{2}$ parametrized through virtuality distribution amplitudes (VDA) $\Phi(x, \sigma)$, with $x$ being Fourier-conjugate to $(p z)$ and $\sigma$ Laplace-conjugate to $z^{2}$. For intervals with $z^{+}=0$, we introduce the transverse momentum distribution amplitude (TMDA) $\Psi\left(x, k_{\perp}\right)$, and write it in terms of VDA $\Phi(x, \sigma)$. We propose models for soft VDAs/TMDAs, and use them for comparison of handbag results with experimental (BaBar and BELLE) data. We also discuss the generation of hard tails of TMDAs from initially soft forms.
\end{abstract}

Keywords: Virtuality distributions; pion transition form factor.

PACS numbers: 11.10.-z, 12.38.-t, 13.60.Fz

\section{Introduction}

Analysis of effects due to parton transverse momentum is an important direction in modern studies of hadronic structure. In particular, cross sections of inclusive processes, such as semi-inclusive deep inelastic scattering (SIDIS) and Drell-Yan pair production are written in terms of transverse momentum dependent distributions (TMDs) $f\left(x, k_{\perp}\right)[1]$, which are generalizations of the usual 1-dimensional parton densities $f(x)$. Within the operator product expansion approach (OPE) $f(x)$ is defined ${ }^{2,3}$ as a function whose $x^{n}$ moments are proportional to matrix elements of twist-2 operators containing $n$ derivatives $\left(D^{+}\right)^{n}$ in the "longitudinal plus" direction. Analogously, the $\left(k_{\perp}^{2}\right)^{l}$ moments of TMDs correspond to matrix elements of

*Notice: Authored by Jefferson Science Associates, LLC under U.S. DOE Contract No. DEAC05-06OR23177. The U.S. Government retains a non-exclusive, paid-up, irrevocable, world-wide license to publish or reproduce this manuscript for U.S. Government purposes.

This is an Open Access article published by World Scientific Publishing Company. It is distributed under the terms of the Creative Commons Attribution 3.0 (CC-BY) License. Further distribution of this work is permitted, provided the original work is properly cited. 
operators containing the derivative $\left(D_{\perp}^{2}\right)^{l}$ in the transverse direction. However, in a usual twist decomposition of an original bilocal operator $\bar{\psi}(0) \ldots \psi(z)$ one deals with Lorentz invariant traces of $\bar{\psi}(0) D_{\mu} \ldots D^{\mu} \psi(0)$ type that correspond to parton distributions in virtuality $k^{2}$ rather than transverse momentum $k_{\perp}^{2}$.

Our goal is to analyze the relationship between distributions in virtuality and distributions in transverse momentum. We start the study with exclusive processes, choosing also the simplest process of $\gamma^{*} \gamma \rightarrow \pi^{0}$ transition that involves just one hadron. In our OPE-type analysis, we encounter a TMD-like object, the transverse momentum dependent distribution amplitude (TMDA) $\Psi\left(x, k_{\perp}\right)$ that is a 3 dimensional generalization of the pion distribution amplitude $\varphi_{\pi}(x) .{ }^{4-7}$ Its definition is similar to that of TMDs $f\left(x, k_{\perp}\right)$, and also to that of the pion wave function used in the standard light-front formalism (see, e.g. Ref. [8]).

\section{Transition form factor in scalar model}

Consider a scalar analog of the handbag contribution to $\gamma\left(q^{\prime}\right) \gamma^{*}(q) \rightarrow \pi^{0}(p)$ form factor $\left(q^{\prime 2}=0, q^{2}=-Q^{2}\right.$, see Fig. 1). In the coordinate representation, we have

$$
T(p, q)=\int d^{4} z e^{-i(q z)} D^{c}(z)\langle p|\phi(0) \phi(z)| 0\rangle,
$$

where $D^{c}(z)=-i / 4 \pi^{2} z^{2}$ is the scalar massless propagator.

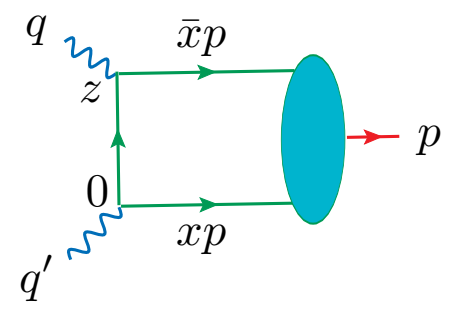

Fig. 1. Handbag diagram in the coordinate representation and parton momentum assignment.

Introducing the twist-2 distribution amplitude $\varphi(x)$

$$
\langle p|\phi(0) \phi(z)| 0\rangle=\int_{0}^{1} \varphi(x) e^{i \bar{x}(p z)} d x+\mathcal{O}\left(z^{2}\right)
$$

gives the twist- 2 approximation ${ }^{8}$ for the transition amplitude

$$
T(p, q)=\left.\int_{0}^{1} \frac{\varphi(x)}{-\left(q^{\prime}-x p\right)^{2}} d x\right|_{p^{2}=0}=\int_{0}^{1} \frac{\varphi(x)}{x Q^{2}} d x .
$$

To go beyond the twist-2 level, one may start with the Taylor expansion

$$
\phi(z)=\sum_{n=0}^{\infty} \frac{1}{n !} z_{\mu_{1}} \ldots z_{\mu_{n}} \partial^{\mu_{1}} \ldots \partial^{\mu_{n}} \phi(0),
$$


and then write $z_{\mu_{1}} \ldots z_{\mu_{n}}$ as a sum of products of powers of $z^{2}$ and symmetrictraceless combinations $\{z \partial\}^{n} \equiv\left\{z_{\mu_{1}} \ldots z_{\mu_{n}}\right\} \partial^{\mu_{1}} \ldots \partial^{\mu_{n}}$. This gives

$$
\phi(z)=\sum_{l=0}^{\infty}\left(\frac{z^{2}}{4}\right)^{l} \sum_{N=0}^{\infty} \frac{N+1}{l !(N+l+1) !}\{z \partial\}^{N}\left(\partial^{2}\right)^{l} \phi(0) .
$$

Assuming that matrix elements of higher twist operators containing powers of $\partial^{2}$ are finite and parametrizing them, for $p^{2}=0$, through

$$
\left.\left\langle p\left|\phi(0)\{z \partial\}^{k}\left(\partial^{2}\right)^{l} \phi(0)\right| 0\right\rangle\right|_{p^{2}=0} \equiv[i(z p)]^{k} \Lambda^{2 l} A_{k l}
$$

(with the size of $\left\langle\partial^{2}\right\rangle$ characterized by some scale $\Lambda^{2}$ ), and then treating the coefficients $A_{k l}$ as $\bar{x}^{k}$ moments of higher-twist DA's $\varphi_{l}(x)$, we arrive at parameterization

$$
\langle p|\phi(0) \phi(z)| 0\rangle=\sum_{l=0}^{\infty}\left(\frac{\Lambda^{2} z^{2}}{4}\right)^{l} \int_{0}^{1} d x \varphi_{l}(x) e^{i \bar{x}(p z)} \equiv \int_{0}^{1} d x B\left(x, z^{2} / 4\right) e^{i \bar{x}(p z)}
$$

in terms of the bilocal function $B\left(x, z^{2} / 4\right)$ accumulating information about parton virtuality. In fact, using the $\alpha$-representation as outlined in Refs. $[9,10,11]$, one can write the contribution of any Feynman diagram to $\langle p|\phi(0) \phi(z)| 0\rangle$ as

$$
\begin{aligned}
& \langle p|\phi(0) \phi(z)| 0\rangle=\mathrm{const} \int_{0}^{\infty} \prod_{j=1}^{l} d \alpha_{j}[A(\alpha)+B(\alpha)]^{-d / 2} \exp \left\{-i \frac{z^{2} / 4}{A(\alpha)+B(\alpha)}\right\} \\
& \quad \times \exp \left\{i(p z) \frac{B(\alpha)}{A(\alpha)+B(\alpha)}\right\} \exp \left\{i p^{2} C(\alpha)-i \sum_{j} \alpha_{j}\left(m_{j}^{2}-i \epsilon\right)\right\},
\end{aligned}
$$

with positive functions $A(\alpha), B(\alpha), C(\alpha)$ of the $\alpha_{\sigma}$-parameters. In other words, without any assumptions about the regularity of the $z^{2} \rightarrow 0$ limit, one can write

$$
\langle p|\phi(0) \phi(z)| 0\rangle=\int_{0}^{\infty} d \sigma \int_{0}^{1} d x \Phi(x, \sigma) e^{i \bar{x}(p z)-i \sigma\left(z^{2}-i \epsilon\right) / 4} .
$$

Moreover, there is no need to assume that $p^{2}=0$ and specify a particular frame. Note also the $z^{2}-i \epsilon$ type dependence and the support property $\sigma \geq 0$.

\section{Transverse momentum dependent distributions}

The parton picture implies a frame in which $p$ has no transverse component and a large component in the "plus" direction. Taking a space-like separation $z$ having $z^{-}$and $z_{\perp}$ components only (i.e., $z^{+}=0$ ), we can introduce the impact parameter distribution amplitude (IDA) $\varphi\left(x, z_{\perp}\right) \equiv B\left(x,-z_{\perp}^{2} / 4\right)$,

$$
\left.\langle p|\phi(0) \phi(z)| 0\rangle\right|_{z^{+}=0, p_{\perp}=0}=\int_{0}^{1} d x \varphi\left(x, z_{\perp}\right) e^{i \bar{x}\left(p z^{-}\right)} .
$$

The IDA function may be treated as a Fourier transform

$$
\varphi\left(x, z_{\perp}\right)=\int \Psi\left(x, k_{\perp}\right) e^{i\left(k_{\perp} z_{\perp}\right)} d^{2} k_{\perp}=\int_{0}^{\infty} d \sigma \Phi(x, \sigma) e^{i \sigma\left(z_{\perp}^{2}+i \epsilon\right) / 4}
$$


of the transverse momentum dependent distribution amplitude (TMDA) $\Psi\left(x, k_{\perp}\right)$. One can "always" write TMDA in terms of VDA as

$$
\Psi\left(x, k_{\perp}\right)=\frac{i}{\pi} \int_{0}^{\infty} \frac{d \sigma}{\sigma} \Phi(x, \sigma) e^{-i\left(k_{\perp}^{2}-i \epsilon\right) / \sigma} .
$$

However, if the $z^{2} \rightarrow 0$ limit is regular (i.e., for soft TMDAs, which vanish for large $k_{\perp}$ faster than any power of $1 / k_{\perp}^{2}$ ), one can also write a relation for $k_{\perp}^{2}$ moments

$$
\int \Psi\left(x, k_{\perp}\right) k_{\perp}^{2 n} d^{2} k_{\perp}=\frac{n !}{i^{n}} \int_{0}^{\infty} \sigma^{n} \Phi(x, \sigma) d \sigma .
$$

In actual calculations, we have no need to pick out integrations over transverse momentum. All the coordinate integrations are done covariantly. The results are expressed in terms of VDA $\Phi(x, \sigma)$, and then Eq. (12) is incorporated that relates $\Phi(x, \sigma)$ to the TMDA $\Psi\left(x, k_{\perp}\right)$. Our final expressions are given in terms of TMDA.

The strategy outlined above may be illustrated by calculation of the Compton amplitude (1). Using the VDA parameterization (9) gives

$$
T\left(Q^{2}\right)=\int_{0}^{1} \frac{d x}{x Q^{2}} \int_{0}^{\infty} d \sigma \Phi(x, \sigma)\left\{1-e^{-\left[i x Q^{2}+\epsilon\right] / \sigma}\right\} .
$$

The first term in the brackets does not depend on $\sigma$ and produces the twist-2 expression (3). Using the TMDA/VDA relation (12) we get

$$
T\left(Q^{2}\right)=\int_{0}^{1} \frac{d x}{x Q^{2}} \int_{k_{\perp}^{2} \leq x Q^{2}} \Psi\left(x, k_{\perp}\right) d^{2} k_{\perp} .
$$

When quarks have spin $1 / 2$, the handbag diagram is given by

$$
\int d^{4} z e^{-i(q z)}\left\langle p\left|\bar{\psi}(0) \gamma^{\nu} S^{c}(-z) \gamma^{\mu} \psi(z)\right| 0\right\rangle=i \epsilon^{\mu \nu \alpha \beta} p_{\alpha} q_{\beta} F\left(Q^{2}\right),
$$

where $S^{c}(z)=\not / 2 \pi^{2}\left(z^{2}\right)^{2}$ is the massless fermion propagator. Using that the antisymmetric part of $\gamma^{\nu} \not \gamma^{\mu}$ is $i z_{\beta} \epsilon^{\mu \nu \alpha \beta} \gamma_{5} \gamma_{\alpha}$ and writing the VDA parameterization

$$
\left\langle p\left|\bar{\psi}(0) \gamma_{5} \gamma_{\alpha} \psi(z)\right| 0\right\rangle=i p_{\alpha} \int_{0}^{\infty} d \sigma \int_{0}^{1} d x \Phi(x, \sigma) e^{i \bar{x}(p z)-i \sigma\left(z^{2}-i \epsilon\right) / 4}+\ldots
$$

we obtain

$$
F\left(Q^{2}\right)=\int_{0}^{\infty} d \sigma \int_{0}^{1} \Phi(x, \sigma) \frac{d x}{x Q^{2}}\left\{1+\frac{i \sigma}{x Q^{2}}\left[1-e^{-\left[i x Q^{2}+\epsilon\right] / \sigma}\right]\right\} .
$$

For large $Q^{2}$, Eq. (18) contains a power-like $\sim 1 / Q^{4}$ correction that corresponds to the twist- $4 \bar{\psi} \gamma_{5} \gamma_{\alpha} D^{2} \psi$ operators. The remaining term contains contributions "invisible" in the OPE. In terms of TMDA we have

$$
F\left(Q^{2}\right)=\int_{0}^{1} \frac{d x}{x Q^{2}} \int_{0}^{x Q^{2}} \frac{d k_{\perp}^{2}}{x Q^{2}} \int_{{k_{\perp}^{\prime}}^{2} \leq k_{\perp}^{2}} \Psi\left(x, k_{\perp}^{\prime}\right) d^{2} k_{\perp}^{\prime} .
$$

In gauge theories, the handbag term in a covariant gauge should be complemented by diagrams corresponding to operators $\bar{\psi}(0) \ldots A\left(z_{i}\right) \ldots \psi(z)$ containing 


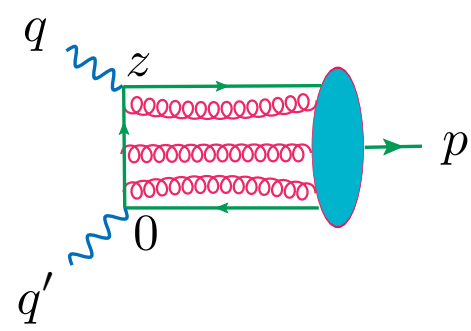

Fig. 2. Structure of the handbag contribution in QCD.

twist-0 gluonic field $A_{\mu_{i}}\left(z_{i}\right)$ inserted into the hard quark propagator (see Fig. 2). It is well known ${ }^{12-14}$ that these insertions may be organized into a path-ordered exponential

$$
E(0, z ; A) \equiv P \exp \left[i g z_{\mu} \int_{0}^{1} d t A^{\mu}(t z)\right]
$$

accumulating the zero-twist field $A^{\mu}$, and insertions of the non-zero twist gluon field

$$
\mathfrak{A}^{\mu}(z)=z_{\nu} \int_{0}^{1} G^{\mu \nu}(s z) s d s
$$

which is the vector potential in the Fock-Schwinger gauge. ${ }^{15,16}$ These insertions correspond to three-body $\bar{q} G q$, etc. higher Fock components. At the two-body $\bar{q} q$ Fock component level, we deal with the gauge-invariant bilocal operator

$$
\mathcal{O}^{\alpha}(0, z ; A) \equiv \bar{\psi}(0) \gamma_{5} \gamma^{\alpha} E(0, z ; A) \psi(z)
$$

Its Taylor expansion has precisely the same structure as that for the original $\bar{\psi}(0) \gamma_{5} \gamma^{\alpha} \psi(z)$ operator, with the only change that one should use covariant derivatives $D^{\mu}=\partial^{\mu}-i g A^{\mu}$ instead of the ordinary $\partial^{\mu}$ ones:

$$
E(0, z ; A) \psi(z)=\sum_{n=0}^{\infty} \frac{1}{n !} z_{\mu_{1}} \ldots z_{\mu_{n}} D^{\mu_{1}} \ldots D^{\mu_{n}} \psi(0) .
$$

This means that one can introduce the parameterization

$$
\begin{aligned}
\left\langle p\left|\mathcal{O}^{\alpha}(0, z ; A)\right| 0\right\rangle= & i p^{\alpha} \int_{0}^{\infty} d \sigma \int_{0}^{1} d x \Phi(x, \sigma) e^{i \bar{x}(p z)-i \sigma\left(z^{2}-i \epsilon\right) / 4} \\
& +z^{\alpha} \ldots
\end{aligned}
$$

that accumulates information about higher twist terms in $\operatorname{VDA} \Phi(x, \sigma)$ and proceed exactly like in a non-gauge case. 


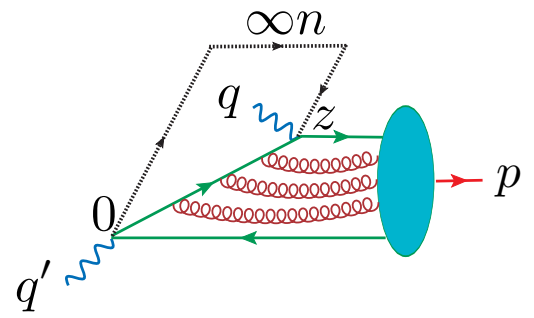

Fig. 3. Organizing twist-0 gluons into a "stapled" link.

Another possibility is to organize the twist-0 gauge fields into a "stapled" link corresponding to a gauge-invariant bilocal operator

$$
\mathcal{O}_{n}^{\alpha}(0, z ; A) \equiv \bar{\psi}(0) E_{n}^{\dagger}(0 ; A) \gamma_{5} \gamma^{\alpha} E_{n}(z ; A) \psi(z)
$$

that involves two infinite-length Wilson lines

$$
E_{n}(z ; A) \equiv P \exp \left[i g \int_{0}^{\infty} d t n_{\mu} A^{\mu}(z+t n) e^{-\epsilon t}\right] .
$$

The accompanying insertions of the non-zero twist gluons are generated then by the field $\mathfrak{A}_{n}^{\mu}(z)$ satisfying the axial gauge condition $n_{\mu} \mathfrak{A}_{n}^{\mu}(z)=0$ and given by

$$
\mathfrak{A}_{n}^{\mu}(z)=n_{\nu} \int_{0}^{\infty} d t G^{\mu \nu}(z+t n) e^{-\epsilon t} .
$$

The Taylor expansion of $\mathcal{O}_{n}^{\alpha}(0, z ; A)$ involves "longer" covariant derivatives $\mathcal{D}^{\mu}=D^{\mu}+i g \mathfrak{A}_{n}^{\mu}$, with the extra term reflecting an additional transverse momentum which is analogous to "lensing" phenomenon" ${ }^{17}$ in the TMD-based description of SIDIS and Drell-Yan processes.

However, it makes no sense to use stapled links in the case of the pion transition form factor. It is easy to show that the insertions of the axial-gauge field $\mathfrak{A}_{n}^{\mu}$ into the hard quark propagator may be combined into a closed contour corresponding to the difference between the straight link and a stapled link (thus restoring the straightlink bilocal operator (22)), with the remaining nonzero-twist gluon insertions given by the Fock-Schwinger field $\mathfrak{A}^{\mu}(z)$.

The use of "stapled" links is natural for processes involving two pions, like $\gamma^{*} \pi\left(p_{1}\right) \rightarrow \pi\left(p_{2}\right)$ (pion EM form factor), where one should take $n=p_{2}$ for $\left\langle 0|\ldots| p_{1}\right\rangle$ and $n=p_{1}$ for $\left\langle p_{2}|\ldots| 0\right\rangle$.

\section{Modeling soft transverse momentum dependence}

Note that Lorentz invariance is fully incorporated in generic VDA representation (9) which treats $(p z)$ and $z^{2}$ as independent variables. Thus, there are no reasons 
for an a priori correlation of $x$ and $\sigma$ dependence in VDA. For simplicity, let us consider factorized models $\Phi(x, \sigma)=\varphi(x) \Phi(\sigma)$ or $\Psi\left(x, k_{\perp}\right)=\varphi(x) \psi\left(k_{\perp}^{2}\right) / \pi$.

It is popular to assume a Gaussian dependence on $k_{\perp}$,

$$
\Psi_{G}\left(x, k_{\perp}\right)=\frac{\varphi(x)}{\pi \Lambda^{2}} e^{-k_{\perp}^{2} / \Lambda^{2}} ; \varphi_{G}\left(x, z_{\perp}\right)=\varphi(x) e^{-z_{\perp}^{2} \Lambda^{2} / 4} .
$$

in this case, the IDA also has a Gaussian dependence on $z_{\perp}$, and one may argue that this fall-off is too fast for large $z_{\perp}$. For comparison, the propagator

$$
D^{c}(z, m)=\frac{1}{16 \pi^{2}} \int_{0}^{\infty} e^{-i \sigma z^{2} / 4-i\left(m^{2}-i \epsilon\right) / \sigma} d \sigma
$$

of a massive particle falls off as $\sim e^{-|z| m}$ for large space-like distances. At small $z^{2}$, however, the free particle propagator has $1 / z^{2}$ singularity while we want a model for $\langle p|\phi(0) \phi(z)| 0\rangle$ that is finite at $z=0$. The simplest way is to add a constant term $\left(-4 / \Lambda^{2}\right)$ to $z^{2}$ in the VDA representation (9). So, we take

$$
\Phi_{m}(x, \sigma)=\varphi(x) \frac{e^{i \sigma / \Lambda^{2}-i m^{2} / \sigma}}{2 i m \Lambda K_{1}(2 m / \Lambda)}, \Psi_{m}\left(x, k_{\perp}\right)=\varphi(x) \frac{K_{0}\left(2 \sqrt{k_{\perp}^{2}+m^{2}} / \Lambda\right)}{\pi m \Lambda K_{1}(2 m / \Lambda)}
$$

as a non-Gaussian model for VDA. To concentrate on the effects of introducing $\Lambda$, one can further take $m=0$, i.e. consider

$$
\Phi_{s}(x, \sigma)=\varphi(x) \frac{e^{i \sigma / \Lambda^{2}-\epsilon \sigma}}{i \Lambda^{2}}, \varphi_{s}\left(x, z_{\perp}\right)=\frac{\varphi(x)}{1+z_{\perp}^{2} \Lambda^{2} / 4} .
$$

Let us now use these models to calculate the transition form factor with the help of Eq.(19). In case of the Gaussian model (28), we have

$$
F_{G}\left(Q^{2}\right)=\int_{0}^{1} \frac{d x}{x Q^{2}} \varphi(x)\left[1-\frac{\Lambda^{2}}{x Q^{2}}\left(1-e^{-x Q^{2} / \Lambda^{2}}\right)\right] .
$$

For large $Q^{2}$, Eq. (32) displays twist-2 and twist-4 contributions, and the term that falls faster than any power of $1 / Q^{2}$. Note that the formal $Q^{2} \rightarrow 0$ limit is finite:

$$
F_{G}\left(Q^{2}=0\right)=\frac{f_{\pi}}{2 \Lambda^{2}}, f_{\pi}=\int_{0}^{1} \varphi(x) d x
$$

In fact, $F\left(Q^{2}\right)$ is finite for $Q^{2}=0$ in any model with finite $\Psi\left(x, k_{\perp}=0\right)$,

$$
F\left(Q^{2}=0\right)=\frac{\pi}{2} \int_{0}^{1} \Psi\left(x, k_{\perp}=0\right) d x .
$$

Using the non-Gaussian model (31) with $m=0$ gives

$$
F\left(Q^{2}\right)=\int_{0}^{1} \frac{d x}{x Q^{2}} \varphi(x)\left[1-\frac{\Lambda^{2}}{x Q^{2}}+2 K_{2}(2 \sqrt{x} Q / \Lambda)\right] .
$$

The size of the twist- 4 term here is given by the confinement scale $\Lambda^{2}$, just as in the Gaussian model. 


\section{Comparison of soft TMDA models with data}

In QCD, the twist-2 approximation for $F\left(Q^{2}\right)$ in the zeroth order in $\alpha_{s}$ is ${ }^{8}$

$$
F^{\mathrm{LOpQCD}}\left(Q^{2}\right)=\int_{0}^{1} \frac{d x}{x Q^{2}} \varphi(x) .
$$

Thus, taking the value of $I\left(Q^{2}\right) \equiv Q^{2} F\left(Q^{2}\right) / f_{\pi}$ from the data one may hope to obtain information about the shape of the pion DA. In particular, for DAs of $\varphi_{r}(x) \sim$ $(x \bar{x})^{r}$ type, one has $I_{r}^{\mathrm{LOpQCD}}\left(Q^{2}\right)=1+2 / r$, i.e. $I^{\text {as }}\left(Q^{2}\right)=3$ for the "asymptotic" wave function $\varphi^{\text {as }}(x)=6 f_{\pi} x \bar{x}$. The most recent data ${ }^{18,19}$ still show a $Q^{2}$ variation of $I\left(Q^{2}\right)$ (see Fig. 4), especially in case of BaBar data ${ }^{18}$ which contain several points with $I\left(Q^{2}\right)$ values well above 3 .

It was argued ${ }^{20,21}$ that BaBar data indicate that the pion DA is close to a flat function $\varphi^{\text {flat }}(x)=f_{\pi}$. The latter corresponds to $r=0$, and pQCD gives $I^{\text {flat }}=\infty$. As shown in Ref. [20], inclusion of transverse momentum dependence of the pion wave function in the light-front formula of Ref. [8] (see also [22]) eliminates the divergence at $x=0$, and one can produce a curve that fits the BaBar data. Similar curves may be obtained within the VDA approach described in the present paper.
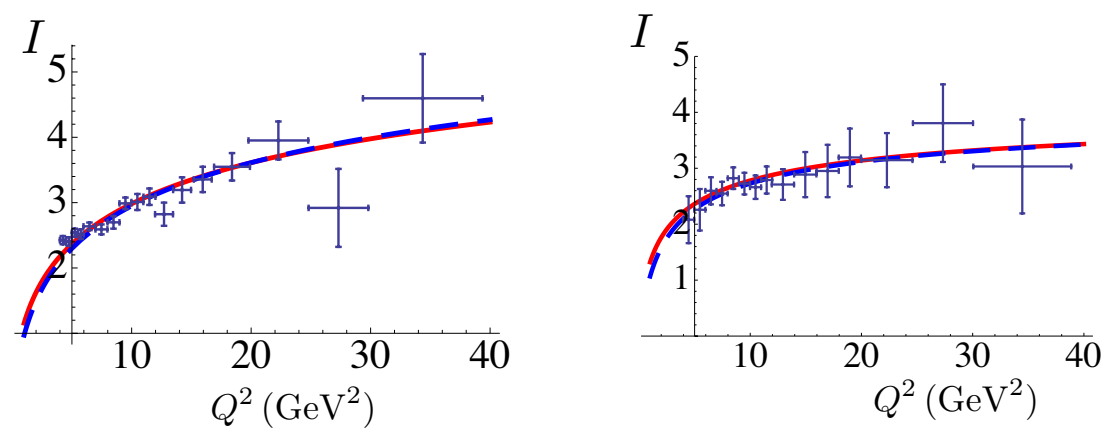

Fig. 4. BABAR (left) and BELLE (right) data compared to model curves described in the text.

In Fig. 4, left, we compare BaBar data with model curves corresponding to flat DA $\varphi(x)=f_{\pi}$ and two types of transverse momentum distributions. First, we take the Gaussian model of Eq. (32). A curve closely following the data is obtained for a value of $\Lambda^{2}=0.35 \mathrm{GeV}^{2}$. For illustration, we also take the non-Gaussian $m=0$ model of Eq. (35), to check what happens in case of unrealistically slow $\sim 1 / z_{\perp}^{2}$ decrease for large $z_{\perp}$. Still, if we take a larger value of $\Lambda^{2}=0.6 \mathrm{GeV}^{2}$, this model produces practically the same curve as the $\Lambda^{2}=0.35 \mathrm{GeV}^{2}$ Gaussian model.

Data from BELLE ${ }^{19}$ give lower values for $I$, suggesting a non-flat DA. In Fig. 4 , right, we show the curves corresponding to $\varphi(x) \sim f_{\pi}(x \bar{x})^{0.4}$ DA. If we take the Gaussian model (32), a good eye-ball fit to data is produced if we take $\Lambda^{2}=0.3 \mathrm{GeV}^{2}$. Practically the same curve is obtained in the non-Gaussian $m=0$ model of Eq. (35) for $\Lambda^{2}=0.4 \mathrm{GeV}^{2}$. 

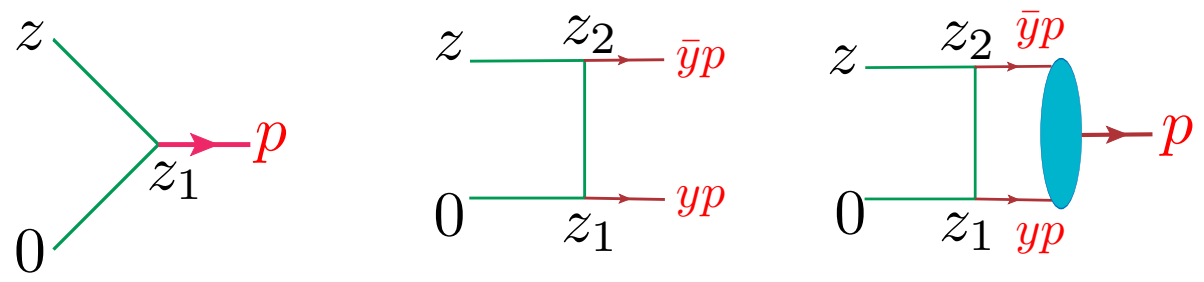

Fig. 5. Modeling VDA by a local current source (left), by a two-current state (middle), and by hard tail model (right).

\section{Modeling hard tail}

Higher-order pQCD corrections also modify the large- $k_{\perp}$ behavior of TMDA, producing a hard $\sim 1 / k_{\perp}^{2}$ tail. Below, we illustrate the basics of using the VDA approach for generation of hard tail terms from original purely soft distributions.

Modeling the matrix element $\langle p|\phi(0) \phi(z)| 0\rangle$ by two propagators $D^{c}\left(z_{1}, m\right)$ and $D^{c}\left(z-z_{1}, m\right)$ (see Fig. 5 , left), with momentum $p$ going out of the point $z_{1}$ gives

$$
\Phi^{\text {point }}(x, \sigma)=\frac{1}{\sigma} e^{i\left(x \bar{x} p^{2}-m^{2}\right) / \sigma}, \Psi^{\text {point }}\left(x, k_{\perp}\right)=\frac{1}{\pi} \frac{1}{k_{\perp}^{2}+m^{2}-x \bar{x} p^{2}}
$$

(after integration over $z_{1}$ ) for the analog of VDA and TMDA, respectively. The resulting TMDA has a hard powerlike $1 / k_{\perp}^{2}$ tail for large $k_{\perp}$. Making a formal $d^{2} k_{\perp}$ integration to produce DA, one faces in this case a logarithmic divergence. In the impact parameter space, we have $\varphi_{2}\left(x, z_{\perp}\right)=2 K_{0}\left(z_{\perp} \sqrt{m^{2}-x \bar{x} p^{2}}\right)$, a function with a logarithmic singularity for $z_{\perp}=0$.

A more complicated toy model involves two currents carrying momenta yp and $(1-y) p \equiv \bar{y} p$ at locations $z_{1}$ and $z_{2}$, respectively (see Fig. 5, middle). With an exchange interaction described by a scalar propagator $D^{c}\left(z_{1}-z_{2}, m\right)$, we have

$$
\Phi^{\operatorname{exch}}(x, \sigma ; y)=i g^{2} \frac{e^{i\left(x \bar{x} p^{2}-m^{2}\right) / \sigma}}{16 \pi^{2} \sigma^{2}} \int_{0}^{\min \left\{\frac{x}{y}, \frac{\bar{x}}{\bar{y}}\right\}} e^{-i y \bar{y} \beta p^{2} / \sigma} d \beta
$$

as an analog of VDA, where $g$ is the coupling constant. For an analog of TMDA in the $p^{2}=0$ limit, we have (using $\alpha_{g} \equiv g^{2} / 16 \pi^{2}$ below)

$$
\Psi^{\mathrm{exch}}\left(x, k_{\perp} ; y\right)=\frac{\alpha_{g}}{\pi} \frac{V(x, y)}{\left(k_{\perp}^{2}+m^{2}\right)^{2}}, V(x, y)=\frac{x}{y} \theta(x<y)+\frac{\bar{x}}{\bar{y}} \theta(x>y),
$$

where $V(x, y)$ is a well-known part of $\mathrm{ERBL}^{5,8}$ evolution kernel.

A further step is to take a superposition of $y p, \bar{y} p$ states with the weight $\varphi_{0}(y)$, a "primordial" distribution amplitude, which results in a convolution model

$$
\Psi^{\mathrm{conv}}\left(x, k_{\perp}\right)=\frac{\alpha_{g}}{\pi} \frac{1}{\left(k_{\perp}^{2}+m^{2}\right)^{2}} \int_{0}^{1} V(x, y) \varphi_{0}(y) d y .
$$

We may further assume that the initial fields at $z_{1}$ and $z_{2}$ are described by some "primordial" bilocal function $B_{0}\left(y,\left(z_{1}-z_{2}\right)^{2} / 4\right)$ corresponding to a soft TMDA 
$\Psi_{0}\left(y, k_{\perp}\right) \equiv \psi_{0}\left(x, k_{\perp}^{2}\right) / \pi$ (see Fig. 5, right). To concentrate on virtuality effects induced by $B_{0}$, we use $m=0$ and $p^{2}=0$, which generates hard TMDA given by

$$
\Psi^{B_{0}}\left(x, k_{\perp}\right)=\frac{\alpha_{g}}{\pi k_{\perp}^{2}} \int_{0}^{1} d y\left[\int_{0}^{1} d \xi \psi_{0}\left(y, \frac{\xi k_{\perp}^{2}}{V(x, y)}\right)\right] .
$$

The term in square brackets may be written as

$$
[\cdots]=\frac{V(x, y)}{k_{\perp}^{2}}\left\{\varphi_{0}(y)-\int_{k_{\perp}^{2} / V(x, y)}^{\infty} \psi_{0}\left(y,{k_{\perp}^{\prime}}^{2}\right) d{k_{\perp}^{\prime}}^{2}\right\},
$$

where $\varphi_{0}(y)$ is the primordial distribution $\Psi_{0}\left(y, k_{\perp}^{\prime}\right)$ integrated over all transverse momenta. Hence, the leading $1 / k_{\perp}^{4}$ hard term is determined by the DA $\varphi_{0}(y)$ only.

In case of spin-1/2 quarks interacting via a (pseudo)scalar gluon field ("Yukawa" gluon model), Eq. (41) is modified by an extra $k_{\perp}^{2}$ factor coming from the numerator spinor trace, which leads to $1 /\left(k_{\perp}^{2}+m^{2}\right)$ dependence in the correction (40) to the TMDA. It results in a $\varphi_{Y}^{\text {conv }}\left(x, z_{\perp}\right) \sim K_{0}\left(m z_{\perp}\right) \delta \varphi(x)$ term for the IDA. The logarithmic divergence for $z_{\perp}=0$ of this outcome corresponds to evolution of the DA. In the $B_{0}$ model, we have (switching $\varphi\left(x, z_{\perp}\right) \rightarrow \varphi\left(x, z_{\perp}^{2}\right)$ below)

$$
\varphi_{Y}^{B_{0}}\left(x, z_{\perp}^{2}\right)=\alpha_{g} \int_{0}^{1} d y V(x, y) \int_{1}^{\infty} \frac{d \nu}{\nu} \varphi_{0}\left(y, \nu z_{\perp}^{2} V(x, y)\right) .
$$

For a function $\varphi_{0}\left(y, z_{\perp}^{2}\right)$ that rapidly decreases when $z_{\perp}^{2} \gtrsim 1 / \Lambda^{2}$, the logarithmically divergent integral over $\nu$ converts into a $\ln \left(z_{\perp}^{2} \Lambda^{2}\right)$ factor accompanying the convolution of $V(x, y)$ and $\varphi_{0}(y)$. This cut-off also results in a finite value

$$
\Psi_{Y}^{B_{0}}\left(x, k_{\perp}=0\right)=\frac{\alpha_{g}}{\pi} \int_{0}^{1} d y \Psi_{0}\left(y, k_{\perp}=0\right)
$$

in the formal $k_{\perp} \rightarrow 0$ limit. Thus, the $\sim 1 / k_{\perp}^{2}$ singularity of the "collinear model" converts into a constant $1 / \Lambda^{2}$ in the Gaussian model.

The full evolution kernel for the pion DA has a plus-prescription form with respect to $x: V(x, y) \rightarrow[V(x, y)]_{+}$. In fact, calculating the $\gamma^{*} \gamma \rightarrow \pi^{0}$ amplitude at $g^{2}$ order, we should include the one-loop vertex and self-energy corrections to the hard quark propagator $S^{c}(z)$. They produce, in particular, a factor of $\ln \left(z^{2}\right)$ multiplying $S^{c}(z)$. We may treat it as a multiplicative modification of VDA convoluted with the original propagator $S^{c}(z)$. This corresponds to adding the $\sim \alpha_{g} \ln \left(z_{\perp}^{2}\right) \varphi_{0}\left(x, z_{\perp}^{2}\right)$ term to $\varphi_{Y}^{B_{0}}\left(x, z_{\perp}^{2}\right)$ as another $\mathcal{O}\left(\alpha_{g}\right)$ correction to IDA.

While the $\sim \ln \left(z_{\perp}^{2}\right)$ behavior for small $z_{\perp}$ is not affected by the choice of the UV renormalization prescription, the large $z_{\perp}$ behavior does. It is convenient to adjust it by taking the Bessel function form $K_{0}\left(z_{\perp} \mu\right)$. Then the correction vanishes for large $z_{\perp}$, and never changes sign. These considerations fix the UV-related correction for IDA to be given by

$$
\varphi_{Y}^{B_{0}, \mathrm{UV}}\left(x, z_{\perp}^{2}\right)=-\alpha_{g} K_{0}\left(z_{\perp} \mu\right) \varphi_{0}\left(x, z_{\perp}^{2}\right) .
$$




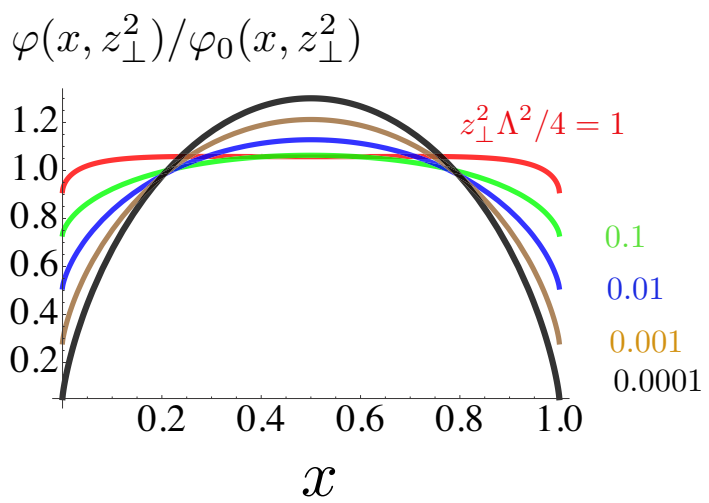

Fig. 6. Evolution for a Gaussian model with flat DA $\varphi_{0}\left(x, z_{\perp}^{2}\right)=e^{-z_{\perp}^{2} / 4 \Lambda^{2}}$ and $\alpha_{g}=0.2$

In Fig. 6 we show the total IDA $\varphi\left(x, z_{\perp}^{2}\right)=\varphi_{0}\left(x, z_{\perp}^{2}\right)+\delta \varphi_{Y}\left(x, z_{\perp}^{2}\right)$ taking for the soft IDA a Gaussian model for the $z_{\perp}$-dependence and flat DA $\varphi_{0}(x)=1$. One can see the change of the $x$-profile from a flat form for large $z_{\perp}$ to asymptotic $\sim x \bar{x}$ for small $z_{\perp}$.

\section{Summary and outlook}

In the present paper, we have outlined a new approach to transverse momentum dependence in hard processes. Its starting point, just like in the OPE formalism, is the use of coordinate representation. At handbag level, the structure of a hadron with momentum $p$ is described by a matrix element of the bilocal operator $\mathcal{O}(0, z)$, treated as a function of $(p z)$ and $z^{2}$. It is parametrized through a virtuality distribution $\Phi(x, \sigma)$, in which the variable $x$ is Fourier-conjugate to $(p z)$, and has the usual meaning of a parton momentum fraction. Another parameter, $\sigma$, is conjugate to $z^{2}$ through an analog of Laplace transform.

Projecting $\mathcal{O}(0, z)$ onto a spacelike interval with $z^{+}=0$, we introduce transverse momentum distributions $\Psi\left(x, k_{\perp}\right)$ and show that they can be written in terms of virtuality distributions $\Phi(x, \sigma)$. This fact opens the possibility to convert the results of covariant calculations, written in terms of $\Phi(x, \sigma)$, into expressions involving $\Psi\left(x, k_{\perp}\right)$. We propose a few simple models for soft VDAs/TMDAs, and use them for comparison of VDA results with experimental (BaBar and BELLE) data on the pion transition form factor.

A natural next step is going beyond the handbag approximation. In QCD, an important feature is that quark-gluon interactions generate a hard $\sim 1 / k_{\perp}^{2}$ tail for TMDAs. To demonstrate the capabilities of the VDA approach in this direction, we have described the basic elements of generating hard tails from soft primordial TMDAs. 
Another direction for future studies is an extension of the VDA approach onto inclusive reactions, such as Drell-Yan and SIDIS processes. In particular, we envisage building VDA-based models for soft parts of TMDs that would have a non-Gaussian behavior at large $k_{\perp}$ (the need for such models was recently emphasized by several authors $^{23-25}$ ). The VDA approach would also allow to self-consistently generate hard tails from these soft TMDs.

\section{Acknowledgments}

I thank I. Balitsky, V.M. Braun, G. A. Miller, A.H. Mueller, A. Prokudin, A. Tarasov and C. Weiss for discussions. This work is supported by Jefferson Science Associates, LLC under U.S. DOE Contract \#DE-AC05-06OR23177 and by U.S. DOE Grant \#DE-FG02-97ER41028.

\section{References}

1. P. Mulders and R. Tangerman, Nucl.Phys. B461, 197 (1996).

2. H. Georgi and H. D. Politzer, Phys.Rev. D14, p. 1829 (1976).

3. A. Efremov and A. Radyushkin, Lett.Nuovo Cim. 19, p. 83 (1977).

4. A. Radyushkin, JINR report P2-10717 (1977).

5. A. Efremov and A. Radyushkin, Phys.Lett. B94, 245 (1980).

6. V. Chernyak, A. Zhitnitsky and V. Serbo, JETP Lett. 26, 594 (1977).

7. G. P. Lepage and S. J. Brodsky, Phys.Lett. B87, 359 (1979).

8. G. P. Lepage and S. J. Brodsky, Phys.Rev. D22, p. 2157 (1980).

9. A. Radyushkin, Phys.Lett. B131, p. 179 (1983).

10. A. Radyushkin, Theor.Math.Phys. 61, p. 1144 (1985).

11. A. Radyushkin, Phys.Rev. D56, 5524 (1997).

12. A. Efremov and A. Radyushkin, JINR report E2-11535 (1978).

13. A. Efremov and A. Radyushkin, Theor.Math.Phys. 44, p. 774 (1981).

14. A. Efremov and A. Radyushkin, Riv.Nuovo Cim. 3N2, 1 (1980).

15. V. Fock, Sowjet. Phys. 12, p. 404 (1937).

16. J. S. Schwinger, Phys.Rev. 82, 664 (1951).

17. M. Burkardt, Phys.Rev. D88, p. 014014 (2013).

18. B. Aubert et al., Phys.Rev. D80, p. 052002 (2009).

19. S. Uehara et al., Phys.Rev. D86, p. 092007 (2012).

20. A. Radyushkin, Phys.Rev. D80, p. 094009 (2009).

21. M. Polyakov, JETP Lett. 90, 228 (2009).

22. I. Musatov and A. Radyushkin, Phys.Rev. D56, 2713 (1997).

23. J. Collins, Int.J.Mod.Phys.Conf.Ser. 25, p. 1460001 (2014).

24. D. Sivers, Int.J.Mod.Phys.Conf.Ser. 25, p. 1460002 (2014).

25. P. Schweitzer, M. Strikman and C. Weiss, JHEP 1301, p. 163 (2013). 